\title{
Cobalt-Tetracyanoquinodimethane Monolayer as Efficient Bi-Functional Single Atom Electrocatalyst: A First Principles Investigation
}

Biswanath Mukherjee ( $\sim$ biswanath.mukh@gmail.com )

Sidho-Kanho-Birsha University https://orcid.org/0000-0001-6140-6088

\section{Research Article}

Keywords: Bi-functionality, electrocatalysis, cobalt-tetracyanoquinodimethane, DFT, first principle calculation, fuel cells

Posted Date: February 17th, 2021

DOI: https://doi.org/10.21203/rs.3.rs-202693/v1

License: (c) (i) This work is licensed under a Creative Commons Attribution 4.0 International License.

Read Full License 


\title{
Cobalt-tetracyanoquinodimethane monolayer as efficient Bi-functional single atom Electrocatalyst: A
}

\section{First Principles Investigation}

\author{
Biswanath Mukherjee* \\ Department of Physics, Sidho-Kanho-Birsha University, Ranchi Road, Purulia, 723104, India \\ *E-mail: biswanath.mukh@gmail.com; biswanath-mukherjee@skbu.ac.in
}

ORCID Id: 0000-0001-6140-6088

\begin{abstract}
A bi-functional electrocatalyst is a stable and catalytically active material for both oxygen reduction reaction (ORR) and oxygen evolution reaction (OER), the development of which is highly challenging, although essential for application in fuel cells. Herein, the first principle based density functional theory (DFT) computations have been carried out to investigate a series of transition metal-tetracyanoquinodimethane (TM-TCNQ: TM = Sc, Ti, V, Cr, Mn, Fe, Co, Ni, $\mathrm{Cu}, \mathrm{Ru}, \mathrm{Rh}, \mathrm{Pd}$ ) monolayer for bi-functional single atom electrocatalyst towards ORR and OER. Amongst various first transition metal row based TCNQ substrates, Cobalt-TCNQ (Co-TCNQ) monolayer was predicted to exhibit best bi-functional catalytic activity. At equilibrium potential (1.23 V, vs RHE), the free energy portraits of Co-TCNQ monolayer for OER in acidic or alkaline media are not completely downhill, measuring an excess potential of $0.6 \mathrm{~V}$, equal to the overpotential, which is needed to be supplied externally to promote the OER activity. On the
\end{abstract}


other hand, an onset potentials of $0.34 \mathrm{~V}$ ( $v s$ RHE) in acidic media are measured for ORR to proceed. The promising catalytic activity of two dimensional Co-TCNQ monolayer towards ORR and OER as revealed by DFT investigations has been explained in terms of density of states and Bader charge analysis.

Keywords: Bi-functionality, electrocatalysis, cobalt-tetracyanoquinodimethane, DFT, first principle calculation, fuel cells.

\section{Introduction}

The consumption of fossil fuel in highly alarming rate and the increasing demand for energy has forced the global researcher to explore the viable alternatives in order to mitigate the crisis in energy. In recent past, fuel cells have been introduced as the novel energy conversion devices $[1,2]$, capable of producing sustained energy through two redox reactions, namely, oxygen reduction reaction (ORR) and oxygen evolution reaction (OER). However, the main bottleneck of ORR being its sluggish reaction kinetics at cathode, which greatly limits the large scale application of fuel cells in practice. Additionally, the platinum (Pt), which has been used so far as highly effective ORR catalyst [3-6], suffers from a number of shortcomings, including, lower abundance, higher cost, and poor durability, which fatally inhibit its wide commercialization [7]. Therefore, the development of non-precious, earth-abundant and non-Pt based ORR electrocatalysts with low overpotential is highly essential for further progress of fuel cell technology. On the other hand, in case of OER, the noble metal oxides, like, ruthenium (Ru) and iridium (Ir) were identified so far to be the best catalyst [8,9], which are not only highly precious, 
but, scarce too. This hinders their widespread scalable applications. Thus, the searching for costeffective and earth abundant electrocatalyst with comparable catalytic performances has become the urgent need. Specially, the designing of stable bi-functional electrocatalyst (ORR and OER) will be a key step towards solution of future energy catastrophe and for development of fuel cell technologies [10].

In recent past, the catalysts based on transition metal (TM)-cored macrocyclic compounds, TM anchored phthalocyanines materials and TM oxides have attracted immense research focus with promising performances [11-20]. The sheet-like structures of these 2-D metal-organic framework offers high electrical conductivity, promoting easy electron transport though the active material and high activity towards single atom electrocatalysts can be obtained through strong anchoring of the metallic centre into the pores [21]. Moreover, the substitution of central metal atom with wide variety of transition metals can modify the molecular structure which can easily tailor the electrochemical functionality of the active catalyst.

Another family of 2-D transition metal-organic molecules, viz, TMtetracyanoquinodimethane (TM-TCNQ), which is similar to the TM- macrocyclic structure, have been explored recently by different groups due to their promising photoelectrochemical and catalytic properties [22-27]. However, the span of diverse activities and the potential of these TM-TCNQ composition till remains unexplored, especially their possible extent as both ORR and OER (bi-functional) electrocatalyst is largely unknown [26]. Most of the earlier studies on various TM-TCNQ framework focussed on the feasibility of these compounds to be applied either as ORR or OER catalyst, which recognised Fe-TCNQ and Ni-TCNQ to be the supreme candidates for ORR and OER respectively, amongst other TM-TCNQ substrates [25-27]. However, the identification of bi-functional catalyst among undoped TM-TCNQ structures so far 
is greatly limited. Earlier, cobalt based composite materials or cobalt doped complex structures have been reported to exhibit promising performances as bi-functional electrocatalysts [28,29]. Experimental investigation on Co-TCNQ complex revealed excellent catalytic activity with overpotential as low as $0.31 \mathrm{~V}$ and long term durability [30]. Also, Co doped graphene substrate in alkaline environment exhibited extremely low overpotential, $c a .0 .21 \mathrm{~V}$, towards OER activity [31], whereas, $\left(\mathrm{Co}(\mathrm{OH})_{2}\right)$ nanocrystals doped with reduced graphene oxide showed an OER overpotential, ca. $0.37 \mathrm{~V}$, which is lower than that of the commercial $\mathrm{RuO}_{2}$ catalyst [32]. Inspired by these reports, density functional calculation has been carried out systematically on various first and second transition metal (TM) row based TCNQ monolayer for applications towards ORR and OER in both acidic and alkaline media.

In this study, first principle calculations on two dimensional transition metaltetracyanoquinodimethane $(\mathrm{TM}-\mathrm{TCNQ}$; TM $=\mathrm{Sc}, \mathrm{Ti}, \mathrm{V}, \mathrm{Cr}, \mathrm{Mn}, \mathrm{Fe}, \mathrm{Co}, \mathrm{Ni}, \mathrm{Cu}, \mathrm{Ru}, \mathrm{Rh}, \mathrm{Pd})$ monolayer were done to investigate their potential application as bi-functional electrocatalyst. Density functional theory (DFT) computations revealed that amongst various TM-TCNQ monolayer, the best performance has been demonstrated by Co-TCNQ substrate surpassing that of the other TM-TCNQ substrates. The Co-TCNQ monolayer revealed overpotential $(\eta)$ value of as low as $c a .0 .6 \mathrm{~V}$ (in both acidic and alkaline media) and onset potential of $0.34 \mathrm{~V}$ ( $v s$ RHE) in acidic media $(\mathrm{pH}=0)$ towards OER and ORR, respectively. The value of $\eta$ for all the TMTCNQ monolayer was found to be independent of electrolyte, i.e., the same value of $\eta$ was obtained in both acidic and alkaline media. DFT computations on Co-TCNQ substrate suggest that strong hybridization between Co-3d with $\mathrm{O}-2 p$ orbitals occurred when $\mathrm{O}_{2}\left(\mathrm{OH}^{*}\right)$ molecule is adsorbed on the Co-TCNQ substrate during ORR (OER). The Bader charge analysis dictated that a net charge of $0.27|e|(0.22|e|)$ has been transferred from Co atom centre towards $\mathrm{O}_{2}$ atom 
$\left(\mathrm{OH}^{*}\right)$ in the Co-TCNQ-OO* (Co-TCNQ-OH*) composite. The promising electrocatalytic performances of Co-TCNQ monolayer shows the potential to overcome the challenges towards the development of cost-effective, earth-abundant, durable and high-efficiency single-atom bifunctional catalyst for the progress of fuel cell technologies as well as for the mitigation of future energy crisis.

\section{DFT calculation methods}

All computations have been carried out with Vienna Ab-initio Simulation Package (VASP) coupled with Projector Augmented Wave (PAW) method. The generalized gradient-corrected functional with Perdew-Burke-Ernzerhof (PBE)-type exchange-correlation potential [33] have been employed in this study. The total energy has been computed using Grimme's DFT-D2 dispersion interactions which includes also Van der Waals correction [34]. For molecular geometry optimization, a $5 \times 5 \times 1$ mesh and for electronic structure calculations, a $11 \times 11 \times 1$ grid have been used. The Bader method was used for the calculation of charge transfer between active sites and the adsorbates. The unit cell of each TM-TCNQ monolayer consists of one metal atom at the centre of the layer, four nitrogen $(\mathrm{N})$ atoms, four hydrogen $(\mathrm{H})$ atoms and twelve carbon (C) atoms. The monolayer was taken parallel to the $x-y$ plane and a vacuum layer of $20 \AA$ thickness is set along $z$-direction between successive TM-TCNQ layers to discard the interlayer interactions. The energy cut-off in the projector augmented wave for the simulation and structure relaxation was set at $550 \mathrm{eV}$ and the convergence threshold for energy and force in the geometry optimization were set respectively as $10^{-5} \mathrm{eV}$ and $0.02 \mathrm{eV} / \AA$. 


\section{Results and Discussions}

The four electron $\left(4 e^{-}\right)$reduction pathway of oxygen $\left(\mathrm{O}_{2}\right)$ consists of adsorption of intermediate products, viz, $\mathrm{OOH}^{*}, \mathrm{O}^{*}$ and $\mathrm{OH}^{*}$ followed by the desorption of $\mathrm{OH}^{-}$. The associative ORR mechanism in acidic environment $(\mathrm{pH}=0)$ proceeds through the following equations:

$$
\begin{gathered}
\mathrm{O}_{2}(g)+* \rightarrow \mathrm{O}_{2}{ }^{*} \\
\mathrm{O}_{2}{ }^{*}+H^{+}+e^{-} \rightarrow O O H^{*} \\
O O H^{*}+H^{+}+e^{-} \rightarrow O^{*}+H_{2} O(\text { liq }) \\
O^{*}+H^{+}+e^{-} \rightarrow O H^{*} \\
O H^{*}+H^{+}+e^{-} \rightarrow H_{2} O(\text { liq })+*
\end{gathered}
$$

where, $(g)$ and (liq) refers respectively to the gas and liquid phase, * represents the active site on the catalyst and $\mathrm{OOH}^{*}, \mathrm{O}^{*}, \mathrm{OH}^{*}$ are the reaction intermediates. The adsorption of $\mathrm{O}_{2}$ on TMTCNQ substrate prefers in most of the cases (except Mn and Cr), the end-on configuration rather than side-on orientation with central metal atom as the favorable adsorption site as obtained from the geometry optimization. On the contrary, $\mathrm{O}_{2}$ adsorption on $\mathrm{Mn}$ - and $\mathrm{Cr}$-TCNQ substrate preferred both end-on and side-on configuration.

Figure 1(a-e) shows the optimized geometries of various ORR intermediates adsorbed on Co-TCNQ monolayer following the above reaction pathway. The adsorption energy $\left(E_{a d}\right)$ of the reaction intermediates on Co-TCNQ surface, summarized in Table 1, has been calculated from the following equation:

$$
E_{a d}=E_{\mathrm{Co}-\mathrm{TCNQ} \text {-adsorbate }}-\left(E_{\mathrm{Co}-\mathrm{TCNQ}}+E_{\text {adsorbate }}\right)
$$


where, $E_{\mathrm{Co}-\mathrm{TCNQ}}$-adsorbate is the energy of the composite system, $E_{\mathrm{Co}-\mathrm{TCNQ}}$ is the energy of the Co-TCNQ monolayer and $E_{\text {adsorbate }}$ is the energy of individual adsorbates. The negative value of $E_{a d}$ would imply favorable adsorption on the catalyst surface. The adsorption energy of $\mathrm{O}_{2}$ molecule on Co-TCNQ substrate for the stable end-on configuration was calculated to be $\sim-0.33$ $\mathrm{eV}$. The $\mathrm{O}-\mathrm{O}$ bond length $\left(d_{O-O}\right)$, which initially was $1.23 \AA$ in isolated $\mathrm{O}_{2}$, found to be increased to the value $1.27 \AA$ when $\mathrm{O}_{2}$ is adsorbed on Co-TCNQ monolayer and forms the composite Co-TCNQ-OO* (Figure 1b). This indicates the possible transfer of electronic charges from central Co-atom to the adsorbed O-atoms in the composite and initiation of ORR mechanism. In the first hydrogenation process, the activated $\mathrm{OO}^{*}$ adsorb a proton $\left(\mathrm{H}^{+}\right)$coupled

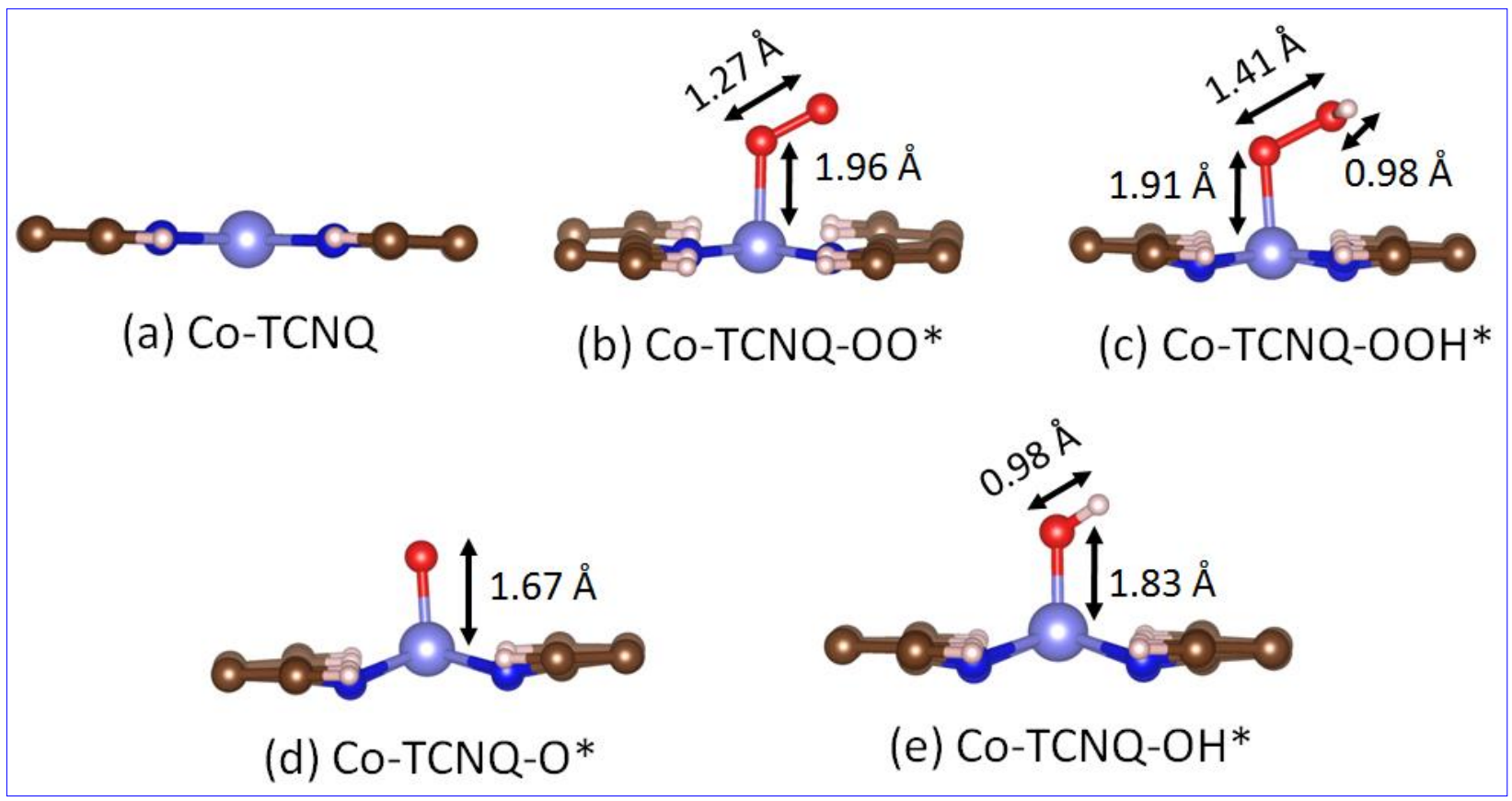

Fig. 1 Optimized geometries of Co-TCNQ structures under different ORR steps.

with an electron (e-) to form $\mathrm{OOH}^{*}$ species. During this step, the $\mathrm{H}$ atom is attached to the upper $\mathrm{O}$ atom and the $\mathrm{O}-\mathrm{O}$ bond length elongated further by $0.14 \AA$ to become $1.41 \AA$ (Figure 1c). Also, the central metal-oxygen bond length $\left(d_{C o-o}\right)$ is calculated to be $c a .1 .96 \AA$ and $1.91 \AA$ during the formation of $\mathrm{OO}^{*}$ and $\mathrm{OOH}^{*}$ adsorbates respectively. During the second 
hydrogenation process, the dissociation of $\mathrm{O}-\mathrm{O}$ bond and subsequent adsorption of $\mathrm{O}^{*}$ on $\mathrm{Co}-$ TCNQ monolayer (Figure 1d) leads to the reduction of $d_{C o-O}$ to the value $1.67 \AA$. In the final reaction step of ORR (Figure 1e), the re-hydrogenation of $\mathrm{O}^{*}$ adsorbate coupled with electron and proton enabled the adsorption of $\mathrm{OH}^{*}$ on the metal binding site and formation of Co-TCNQ$\mathrm{OH}^{*}$. The feasibility of occurring of these steps can further be explained by calculating the Gibbs Free energy of each reaction steps as discussed below.

Table 1: Adsorption energies of different reaction intermediates and various bond lengths on Co-TCNQ monolayer during ORR.

\begin{tabular}{|c|c|c|c|}
\hline Adsobates & $E_{a d}(\mathrm{eV})$ & $d_{C o-O}(\AA)$ & $d_{O-O}(\AA)$ \\
\hline $\mathrm{OO}^{*}$ & -0.33312 & 1.96881 & 1.27094 \\
\hline $\mathrm{OOH}^{*}$ & -2.17726 & 1.91101 & 1.41242 \\
\hline $\mathrm{O}^{*}$ & -3.14303 & 1.67037 & - \\
\hline $\mathrm{OH}^{*}$ & -1.98312 & 1.83417 & - \\
\hline
\end{tabular}

The Gibbs Free energy $(\Delta \mathrm{G})$ for each reaction intermediates during ORR have been calculated using the computational hydrogen electrode model, introduced by Nørskov [35].

$$
\Delta G=\Delta E+\Delta Z P E-T \Delta S-n e U+\Delta G_{p H}
$$

where, $\Delta E$ is the reaction energy as obtained from DFT calculation, $\triangle Z P E$ and $\triangle S$ are respectively the change in zero point energy and entropy upon adsorption which could be estimated from the vibrational frequencies for the adsorbates [36], $n$ is the number of electron transferred, $U$ is the external potential, $\mathrm{T}=298.15 \mathrm{~K}$ is system temperature and $\Delta G_{p H}=$ $2.303 K_{B} T \times p H$ corresponds to the contribution due to $\mathrm{H}^{+}$concentration variation. The adsorption of $\mathrm{O}_{2}$ molecule on the Co-TCNQ substrate is energetically favorable, as it shows 
downhill steps in the free energy diagram in ORR. As shown in Figure 2(a), all the reaction intermediate steps are exothermic at $\mathrm{U}=0 \mathrm{~V}$. However, at equilibrium electrode potential $(\mathrm{U}=$ $1.23 \mathrm{~V}$, vs RHE), the $\mathrm{OOH}^{*}$ steps become endothermic by $0.89 \mathrm{~V}$ and represents the rate determining step. The onset potential estimated from this plot was $0.34 \mathrm{~V}(1.23-0.89 \mathrm{~V})$ which is the maximum limit of the potential up to which the steps remain exothermic.
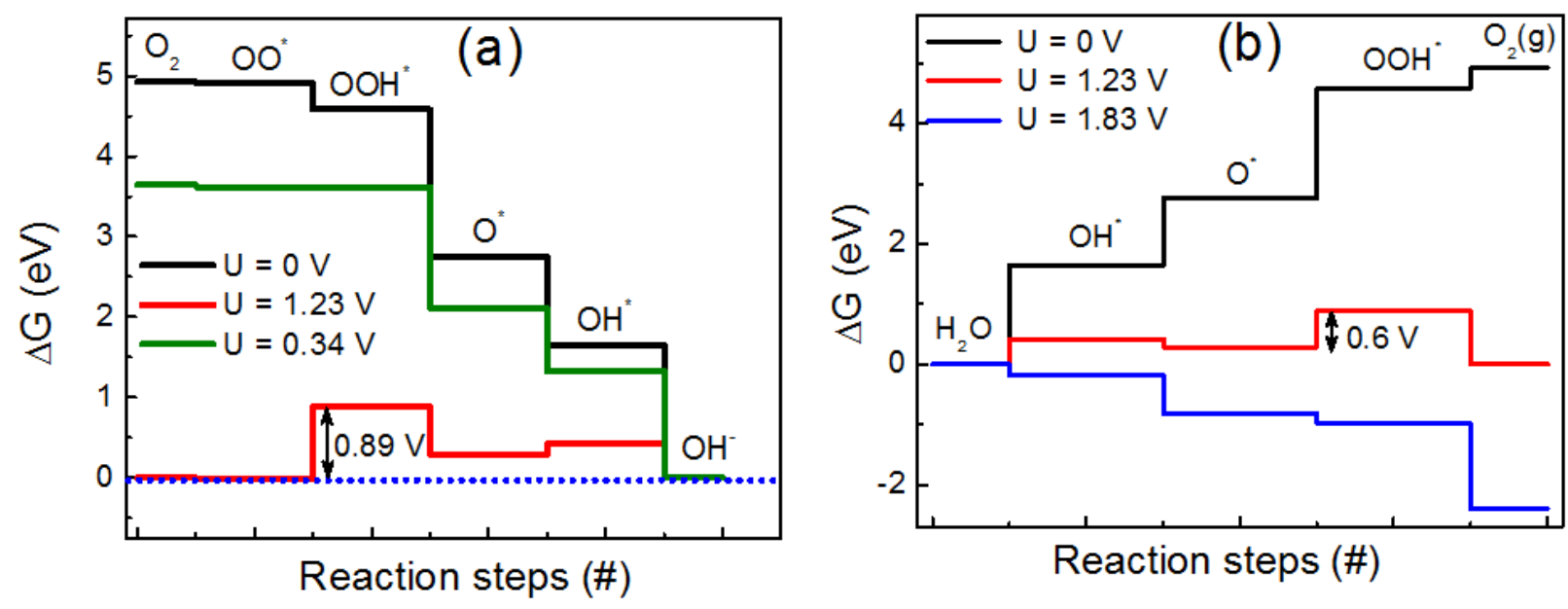

Fig. 2 Gibbs free energy profile for Co-TCNQ catalyst in acidic medium ( $\mathrm{pH}=0$ ) during (a) ORR and (b) OER.

To investigate about OER, which is the reverse reaction of ORR, the adsorbed free energies of various intermediates species $\left(\Delta G_{O H^{*}}, \Delta G_{O^{*}}, \Delta G_{O O H^{*}}\right)$ on Co-TCNQ monolayer were estimated. The free energy profile of Co-TCNQ monolayer towards OER under acidic environment has been depicted in Figure 2(b). At $U=1.23 \mathrm{~V}\left(v s\right.$ RHE), the reaction steps $\mathrm{OH}^{*}$ and $\mathrm{OOH}^{*}$ are endothermic and are thermodynamically unfavorable. Hence additional potential equal to the overpotential $(\eta)$ will be needed to promote these reaction steps. As illustrated in Figure 2(b), the $\mathrm{OOH}^{*}$ formation step is maximum uphill, $c a .0 .6 \mathrm{~V}$ over the equilibrium potential which gives an estimation of $\eta=0.6 \mathrm{~V}$ towards OER for Co-TCNQ based catalyst. Therefore, with additional electrode potential of $0.6 \mathrm{~V}$ over the equilibrium value, i.e., for $U=$ $1.83 \mathrm{~V}$, all reaction steps become downhill to drive the OER process spontaneously. 


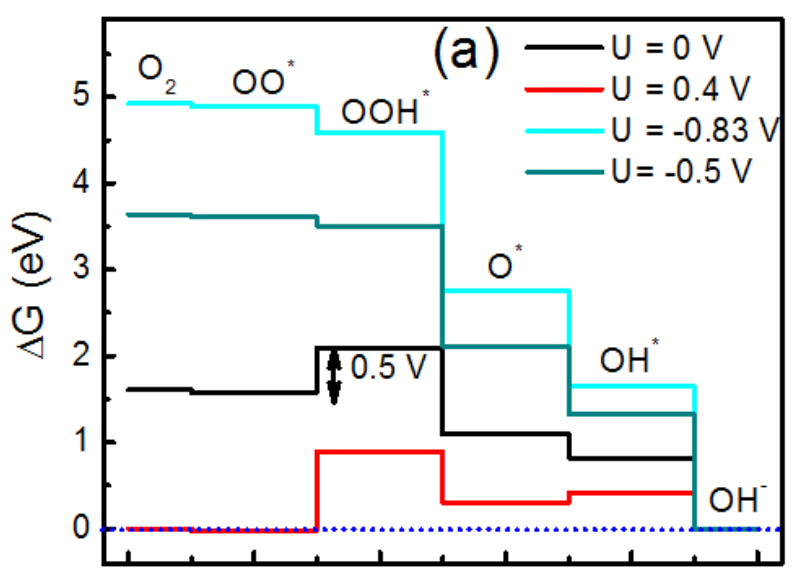

Reaction steps (\#)

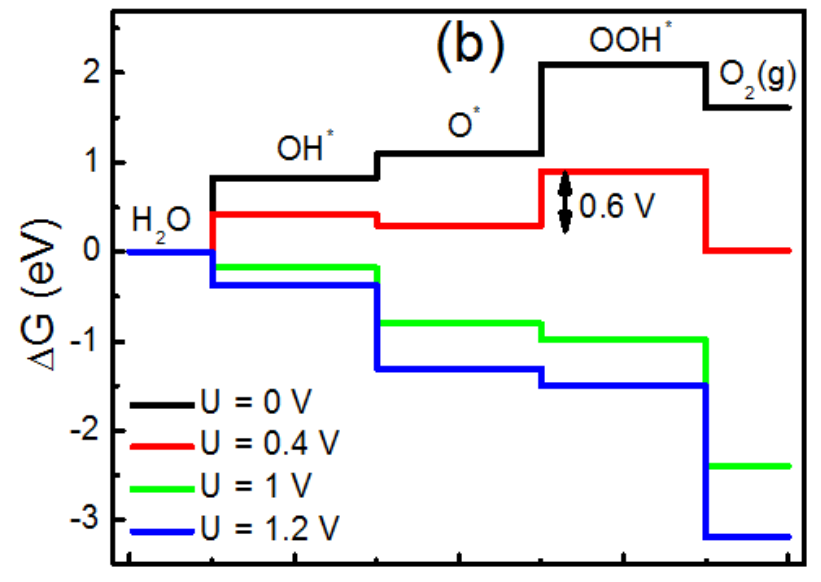

Reaction steps (\#)

Fig. 3 Gibbs free energy profile for Co-TCNQ catalyst in alkaline medium $(\mathrm{pH}=14)$ during (a) ORR and (b) OER.

In alkaline medium $(\mathrm{pH}=14)$, on the other hand, the proton donor is $\mathrm{H}_{2} \mathrm{O}$ and the corresponding equations describing ORR steps will be as follows:

$$
\begin{gathered}
\mathrm{O}_{2}(\mathrm{~g})+* \rightarrow \mathrm{O}_{2}{ }^{*} \\
\mathrm{O}_{2}{ }^{*}+\mathrm{H}_{2} \mathrm{O}(\mathrm{liq})+e^{-} \rightarrow \mathrm{OOH}^{*}+\mathrm{OH}^{-} \\
\mathrm{OOH}^{*}+e^{-} \rightarrow \mathrm{O}^{*}+\mathrm{OH}^{-} \\
\mathrm{O}^{*}+\mathrm{H}_{2} \mathrm{O}(\mathrm{liq})+e^{-} \rightarrow \mathrm{OH}^{*}+\mathrm{OH}^{-} \\
O H^{*}+e^{-} \rightarrow O H^{-}+*
\end{gathered}
$$

The corresponding free energy profile of ORR and OER at different potential have been depicted in Figure 3(a) and (b) respectively. Here, at $\mathrm{U}=0 \mathrm{~V}$, the $\mathrm{OOH}^{*}$ step formation is maximum uphill and is the rate determining step in ORR (Figure 3a). The onset potential estimated from the graph at $\mathrm{U}=0 \mathrm{~V}$ for Co-TCNQ monolayer is $0.5 \mathrm{~V}$ under alkaline condition $(\mathrm{pH}=14)$. 
Finally, at $\mathrm{U}=-0.5 \mathrm{~V}$, all the steps became downhill in the free energy diagram indicating spontaneous ORR reaction. The free energy profile of Co-TCNQ catalyst for OER, depicted in Figure $3 \mathrm{~b}$, illustrates that at equilibrium potential $(\mathrm{U}=0.4 \mathrm{~V})$, the $\mathrm{OOH}^{*}$ formation step is endothermic by $0.6 \mathrm{~V}$. The overpotential value as deduced from the OER free energy profile was $0.6 \mathrm{~V}$, same as obtained under acidic environment. As a result, at $\mathrm{U}=1 \mathrm{~V}$, all the steps in OER become endothermic allowing all reaction intermediates in OER to proceed spontaneously.

To compare the bi-functional catalytic performance of Co-TCNQ monolayer with that of other TM-TCNQ substrates, the DFT calculations have also been carried out for $\mathrm{Sc}, \mathrm{Ti}, \mathrm{V}, \mathrm{Cr}$, $\mathrm{Mn}, \mathrm{Fe}, \mathrm{Ni}, \mathrm{Cu}, \mathrm{Ru}, \mathrm{Rh}$, and Pd based TCNQ substrates. The calculated overpotential $(\eta)$ value towards OER under acidic $(\mathrm{pH}=0)$ condition and the limiting potential $\left(\eta_{\text {lim }}\right)$ towards ORR in alkaline conditions $(\mathrm{pH}=14)$ have been plotted in Figure 4. It is seen from the figure that for TM like $\mathrm{Sc}, \mathrm{Ti}, \mathrm{V}, \mathrm{Cr}$ and $\mathrm{Mn}$, the overpotential is higher than $1 \mathrm{~V}$ and hence is unsuitable to promote any energy efficient OER activity. In general, $\eta$ decreases from left to rightward for first transition metal row (except $\mathrm{Cu}$ ) in the periodic table following the order, $\mathrm{Sc}<\mathrm{V}<\mathrm{Ti}<\mathrm{Cr}<\mathrm{Mn}$ $<\mathrm{Fe}<\mathrm{Co}<\mathrm{Ni}$, and the lowest $\eta$ is obtained for $\mathrm{Ni}(0.48 \mathrm{~V} v s \mathrm{RHE})$ followed by Co $(0.6 \mathrm{~V} v s$ RHE) substrate. However, because of low limiting potential $\left(\eta_{\text {lim }}\right)$ of Ni-TCNQ monolayer (0.18 V vs RHE), it is found to be ineffective as ORR catalyst. The comparable performances as bi-functional catalyst has been observed for Co-TCNQ and Fe-TCNQ monolayer with marginal differences in their $\eta$ and $\eta_{\text {lim }}$ values which indicates that these two monolayer might be the best choice for both OER and ORR catalysts. Nevertheless, many studies have been conducted to evaluate the ORR and OER activities of Fe-TCNQ based catalyst [26,27], but, in depth report on Co-TCNQ electrocatalyst, especially as bi-functional catalyst is highly limited. We, therefore, will focus here on the bi-functional catalytic performances of Co-TCNQ monolayer 


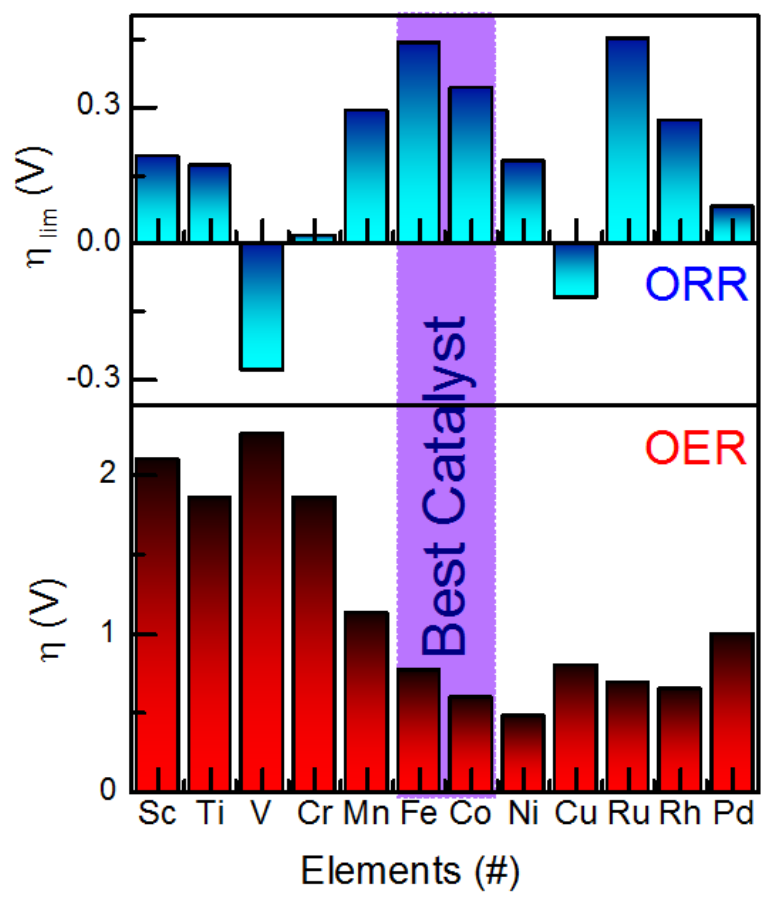

Fig. 4 Bi-functional catalytic performances of various TM-TCNQ monolayer. The limiting potential $\left(\eta_{\text {lim }}\right)$ towards ORR (upper panel) and overpotential $(\eta)$ towards OER (lower panel) has been plotted for different TM-TCNQ monolayer. The best performance zone is marked by violet color.

towards ORR and OER.

To further assess the charge transfer mechanism in Co-TCNQ-OO* composite due to the interlayer interaction during ORR, the difference in charge density $(\Delta \rho)$ between Co-TCNQOO* composite and the individual components, viz, Co-TCNQ monolayer and OO* adsorbate was calculated, i.e.,

$$
\Delta \rho=\rho_{\mathrm{Co}-\mathrm{TCNQ}-\mathrm{OO}^{*}}-\rho_{\mathrm{Co}-\mathrm{TCNQ}}-\rho_{\mathrm{OO}^{*}}
$$




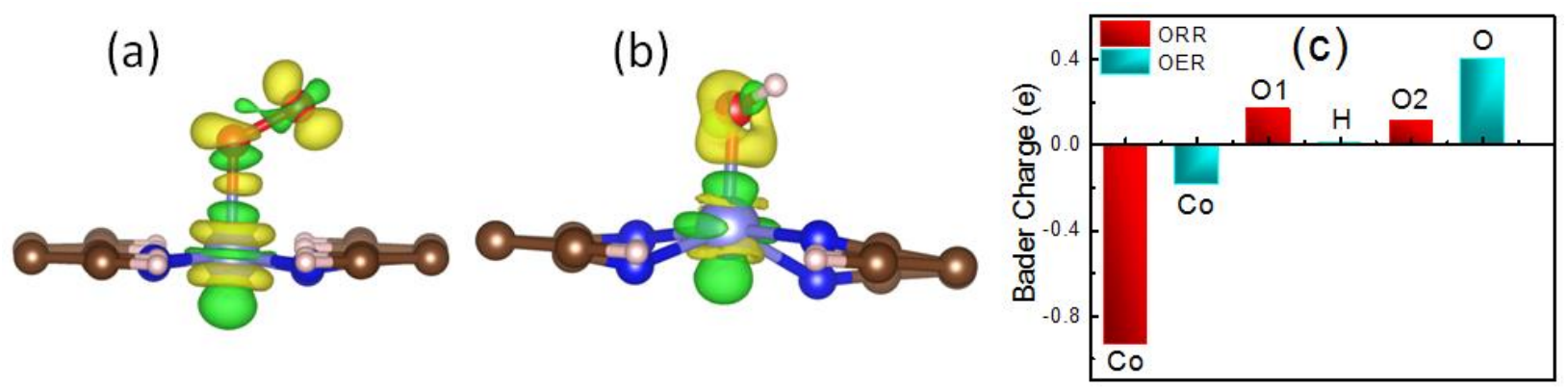

Fig. 5 Charge re-arrangement and corresponding optimized geometries of Co-TCNQ monolayer during (a) OO* and (b) $\mathrm{OH}^{*}$ adsorbates. The yellow and green colors represent respectively the accumulation (negative) and depletion (positive) of charges. (c) Bader charge analysis for both ORR and OER.

The molecular geometry of Co - TCNQ $-00^{*}$ composite under optimized condition along with the charge re-arrangement in different atoms is presented in Figure 5(a). It is observed that depletion of charges occurred in central Co atom (green), and net charge accumulation (yellow) took place around the adsorbate, i.e., O-atoms. To extract the actual charge arrangement in CoTCNQ-OO* composite due to the interaction between substrate and the adsorbate, i.e., between central Co-core and $\mathrm{OO}^{*}$, Bader charge analysis have further been carried out. The summarized results are presented in Figure 5(c). It is seen that the metallic centre is susceptible to lose electrons, while two O-atoms in $\mathrm{OO}^{*}$ adsorbate are more inclined towards gaining of electrons. As a result, a net $0.27|e|$ charges migrated from the metal atom $(\mathrm{Co})$ centre to the $\mathrm{O}$-atoms, which results in the elongation of $\mathrm{O}-\mathrm{O}$ bond length from $1.23 \AA$ to $1.27 \AA$ in $\mathrm{Co}-\mathrm{TCNQ}-00^{*}$ composite and favors the OO* adsorption on the Co-TCNQ substrate to facilitate ORR. In case of OER, the charge re-arrangement configuration and the Bader charge analysis are depicted respectively in Figure 5(b) and 5(c). In this case also, the similar Bader charge calculation resulted in a net charge migration of $0.22|e|$ from Co-atom centre to the $\mathrm{OH}^{*}$ adsorbate in Co TCNQ $-\mathrm{OH}^{*}$ composite. 

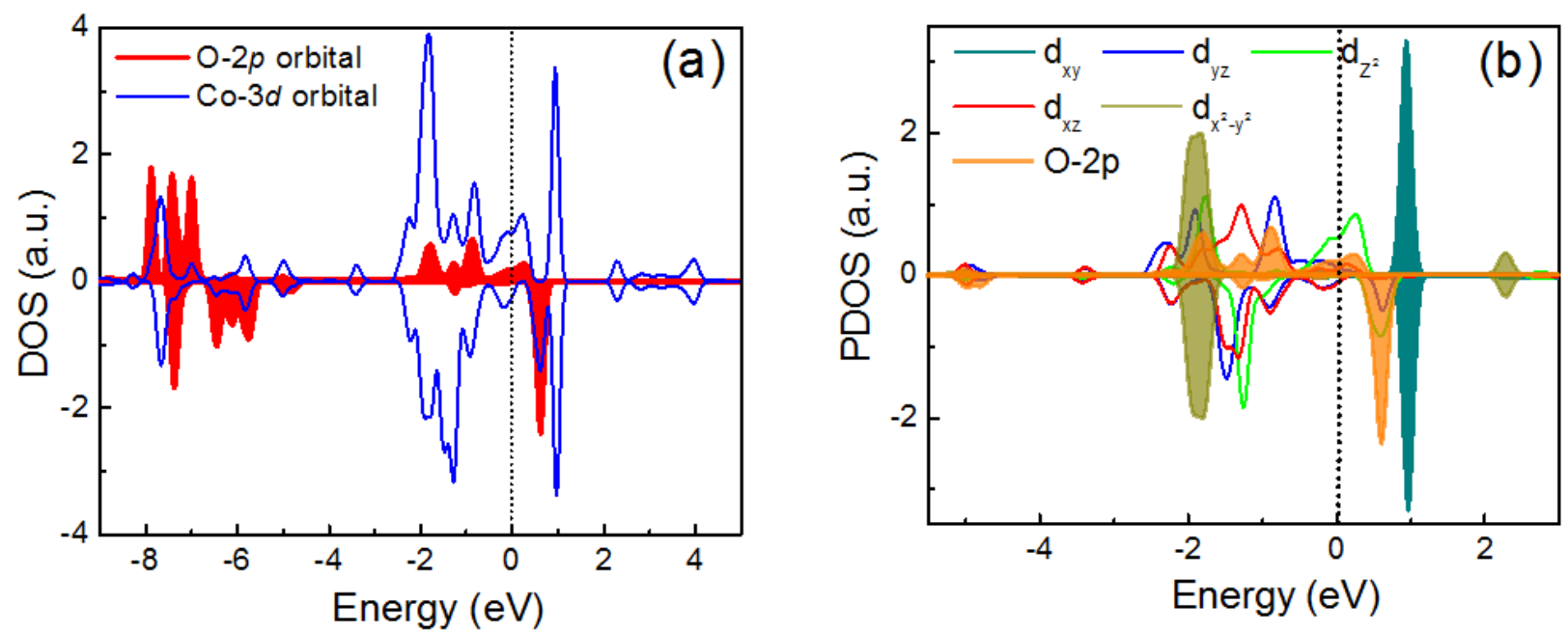

Fig. 6 (a) Density of states (DOS) and hybridization between Co-3d and O-2p orbitals. (b) PDOS of O-2p orbital and different Co-3d orbitals. The dotted lines in the figures at $\mathrm{E}=0 \mathrm{eV}$ indicate the Fermi level.

The electronic structure and the local density of states at the surface of the catalyst plays an important role for adsorption of atoms on the substrate. To get an understanding on the activation of $\mathrm{O}_{2}$ on central Co-atom active site during ORR, the spin polarized density of states (PDOS) have been calculated for Co-TCNQ monolayer under $\mathrm{O}_{2}$ adsorption, the results of which have been portrayed in Figure 6(a). The semi-metallic nature of Co-TCNQ monolayer promotes easy electron transport through the 2-dimensional surface. Moreover, strong hybridization between Co-3d orbital and O-2p orbital has been observed to occur (Figure 6a), which upholds spurious charge transfer between the highly active partially occupied d-orbital of central Coatom to the half filled $\pi_{\mathrm{p}}{ }^{*}$ orbital of $\mathrm{O}_{2}$ atom. To further recognize the contribution towards catalytic activity from five degenerate $3 d$ orbitals of Co-atom, viz, $d_{x^{2}-y^{2}}, d_{z^{2}}, d_{x y}, d_{x z}$, and $d_{y z}$ orbitals, PDOS of each of these has been computed separately. The results are summarized in Figure 6(b). One of the in-plane Co-3d orbitals, $d_{x y}$, are highly sharp in both spin-up and spindown channel and are almost fully empty with no hybridization $\mathrm{O}-2 p$ orbital, while another in- 
plane orbital, $d_{x^{2}-y^{2}}$ is less sharp, but fully filled in both spin-up and spin-down channel with partial hybridization with O- $2 p$ orbital. On the other hand, all out-plane orbitals, $d_{z^{2}}, d_{x z}$, and $d_{y z}$ are fully filled, and are not localized sharp peaks due to the hybridization with O- $2 p$ orbital. The excellent hybridization between $\mathrm{Co}-3 d_{z^{2}}$ with O-2p orbitals in the PDOS in up and down spin channel indicates that dominant role is played by $d_{z^{2}}$ orbital in activating OO* adsorbate on Co-TCNQ monolayer and in promoting the catalytic activity.

\section{Conclusions}

In summary, an energy efficient electrocatalyst based on Co-TCNQ monolayer has been explored for both oxygen reduction reaction and oxygen evolution reaction. Density functional theory computations revealed that amongst various transition metal-TCNQ based monolayer catalyst, Co-TCNQ monolayer has the best potential to serve as bi-functional catalyst towards ORR and OER in acidic or alkaline media. An overpotential of $0.6 \mathrm{~V}$ ( $v s$ RHE) was calculated for the Co-TCNQ catalyst to promote the OER activity in either environment, whereas, onset potentials of $0.34 \mathrm{~V}$ ( $v s$ RHE) in acidic media were measured for ORR to proceed. The other TM-TCNQ based catalyst surfaces have been predicted to exhibit inferior performances in terms of limiting potential and overpotential to drive the ORR and OER processes, respectively. The two-dimensional sheet-like surface of Co-TCNQ monolayer along with its semi-metallic nature in electronic properties helps promoting easy electron transport through the surface and the strong hybridization between Co-3d orbital with O-2p orbital facilitates activation of $\mathrm{O}_{2}$ on CoTCNQ surface for excellent catalytic activity. Bader charge analysis revealed a net transfer of $0.27|\mathrm{e}|$ charge from Co centre to the $\mathrm{OO}^{*}$ adsorbate during ORR and $0.22|\mathrm{e}|$ charge from central 
Co-atom to $\mathrm{OH}^{*}$ adsorbate during OER. The promising bi-functional catalytic activity of Co-TCNQ monolayer might boost up future research to design for two dimensional, durable, earth-abundance and non-precious electrocatalyst for fuel cell and alternative energy technology.

Funding: Not applicable

Conflicts of interest: The author declares no potential conflict of interest.

Availability of data and material (data transparency): Not applicable

Code availability (software application or custom code): Not applicable

\section{References}

[1] Antolini E (2007) Catalysts for direct ethanol fuel cells. J. Power Sources 170: 1-12.

[2] Lamy C, Lima A, LeRhun V, Delime F, Coutanceau C, Léger JM (2002) Recent advances in the development of direct alcohol fuel cells (DAFC). J. Power Sources 105: 283-296.

[3] Debe MK (2012) Electrocatalyst approaches and challenges for automotive fuel cells. Nature 486: 43-51.

[4] Qu J, Ye F, Chen D, Feng Y, Yao Q, Liu H, Xie J, Yang J (2016) Platinum-based heterogeneous nanomaterials via wet-chemistry approaches toward electrocatalytic applications. Adv Colloid Inter Sci 230: 29-53. 
[5] Chen A, Hindle PH (2010) Platinum-Based Nanostructured Materials: Synthesis, Properties, and Applications. Chem Rev 110(6): 3767-3804.

[6] Wu J, Yang H (2013) Platinum-Based Oxygen Reduction Electrocatalysts. Acc Chem Res 46(8): 1848-1857.

[7] Vesborg PCK, Jaramillo TF (2012) Addressing the terawatt challenge: scalability in the supply of chemical elements for renewable energy. RSC Adv 2: 7933-7947.

[8] Reier T, Oezaslan M, Strasser P (2012) Electrocatalytic Oxygen Evolution Reaction (OER) on Ru, Ir, and Pt Catalysts: A Comparative Study of Nanoparticles and Bulk Materials. ACS Catal 2(8): 1765-1772.

[9] Suen NT, Hung SF, Quan Q, Zhang N, Xu YJ, Chen HM (2017) Electrocatalysis for the oxygen evolution reaction: recent development and future perspectives. Chem Soc Rev 46: 337365.

[10] Huang ZF, Wang J, Peng Y, Jung CY, Fisher A, Wang X (2017) Design of Efficient Bifunctional Oxygen Reduction/Evolution Electrocatalyst: Recent Advances and Perspectives. Adv Energy Mater 7: 1700544-1700564.

[11] Jiang Z, Wang Y, Zhang X, Zheng H, Wang X, Liang Y (2019) Revealing the hidden performance of metal phthalocyanines for $\mathrm{CO} 2$ reduction electrocatalysis by hybridization with carbon nanotubes. Nano Res 12: 2330-2334.

[12] Zagal J, Páez M, Tanaka AA, Santos JR, Linkous CA (1992) Electrocatalytic activity of metal phthalocyanines for oxygen reduction. J. Electroanal Chem 339: 13-30. 
[13] Dai L, Xue Y, Qu L, Choi HJ, Baek JB (2015) Metal-Free Catalysts for Oxygen Reduction Reaction. Chem Rev 115: 4823-4892.

[14] Deng D, Novoselov KS, Fu Q, Zheng N, Tian Z, Bao X (2016) Catalysis with twodimensional materials and their heterostructures. Nat Nanotechnol 11: 218-230.

[15] Mukherjee B (2018) Investigation of FePc Nanoribbon as ORR Catalyst in Alkaline

Medium: A DFT Based Approach. J Electrochem Soc 165(15): J3231-J3235.

[16] Mukherjee B (2020) Solvothermally Synthesized Iron Phthalocyanine Nanostructure for High ORR Response: A Joint Experimental Investigation and DFT Analysis. J Electrochem Soc 167: 116501-116507.

[17] Higgins D, Zamani P, Yu A, Chen Z (2016) The application of graphene and its composites in oxygen reduction electrocatalysis: a perspective and review of recent progress. Energy Environ Sci 9: 357-390.

[18] Zhao Z, Li M, Zhang L, Dai L, Xia Z (2015) Design Principles for Heteroatom-Doped Carbon Nanomaterials as Highly Efficient Catalysts for Fuel Cells and Metal-Air Batteries. Adv Mater 27(43): 6834-6840.

[19] Zhou M, Wang HL, Guo S (2016) Towards high-efficiency nanoelectrocatalysts for oxygen reduction through engineering advanced carbon nanomaterials. Chem Soc Rev 45: 1273-1307.

[20] Zhou R, Jaroniec M, Qiao SZ (2015) Nitrogen-Doped Carbon Electrocatalysts Decorated with Transition Metals for the Oxygen Reduction Reaction. ChemCatChem 7: 3808-3817. 
[21] Mao X, Ling C, Tang C, Yan C, Zhu Z, Du A (2018) Predicting a new class of metalorganic frameworks as efficient catalyst for bi-functional oxygen evolution/reduction reactions. J Catal 367: 206-211.

[22] Nafady A, O'Mullane AP, Bond AM (2014) Electrochemical and photochemical routes to semiconducting transition metal-tetracyanoquinodimethane coordination polymers. Coord Chem Rev 268: 101-142.

[23] Ma Y, Dai Y, Wei W, Yu L, Huang B (2013) Novel Two-Dimensional Tetragonal Monolayer: Metal-TCNQ Networks. J Phys Chem A 117(24): 5171-5177.

[24] Zhu G, Sun Q (2016) Recent advances in computational studies of organometallic sheets: Magnetism, adsorption and catalysis. Comput. Mater. Sci. 112: 492-502.

[25] Mukherjee B (2021) Highly Efficient Electrocatalyst for Oxygen Evolution Reaction: DFT Investigation on Transition Metal-Tetracyanoquinodimethane Monolayer. ChemistrySelect 6(4): 609-616.

[26] Deng Q, Zhao J, Wu T, Chen G, Hansen HA, Vegge T (2019) 2D transition metal-TCNQ sheets as bifunctional single-atom catalysts for oxygen reduction and evolution reaction (ORR/OER). J Catal 370: 378-384.

[27] Wang N, Feng L, Shang Y, Zhao J, Cai Q, Jin P (2016) Two-dimensional irontetracyanoquinodimethane (Fe-TCNQ) monolayer: an efficient electrocatalyst for the oxygen reduction reaction. RSC Adv 6: 72952-72958. 
[28] Maruthapandian V, Mathankumar M, Saraswathy V, Subramanian B, Muralidharan S (2017) Study of the Oxygen Evolution Reaction Catalytic Behavior of $\mathrm{Co}_{x} \mathrm{Ni}_{1-\mathrm{x}} \mathrm{Fe}_{2} \mathrm{O}_{4}$ in Alkaline Medium. ACS Appl Mater Interfaces 9(15): 13132-13141.

[29] Zhang W, Cui L, Liu J (2020) Recent advances in cobalt-based electrocatalysts for hydrogen and oxygen evolution reactions. J Alloys Compd 821: 153542-153562.

[30] Wei Y, Ren X, Ma H, Sun X, Zhang Y, Kuang X, Yan T, Wu D, Wei Q (2018) In situ Formed $\mathrm{Co}(\mathrm{TCNQ})_{2}$ Metal-Organic Framework Array as a High-Efficiency Catalyst for Oxygen Evolution Reactions. Chem Eur J 24: 2075-2079.

[31] Zhang Q, Duan Z, Li M, Guan J (2020) Atomic cobalt catalysts for the oxygen evolution reaction. Chem Commun 56: 794-797.

[32] Park SW, Shin HJ, Kim DW (2019) S,N co-doped reduced graphene oxide sheets with cobalt hydroxide nanocrystals for highly active and stable bifunctional oxygen catalysts. Inorg Chem Front 6: 3501-3509.

[33] Perdew JP, Burke K, Ernzerhof M (1996) Generalized Gradient Approximation Made Simple. Phys Rev Lett 77: 3865-3868.

[34] Grimme S (2006) Semiempirical GGA-type density functional constructed with a long-range dispersion correction. J Comput Chem 27(15): 1787-1799.

[35] Peterson AA, Pedersen FA, Studt F, Rossmeisl J, Norskov JK (2010) How copper catalyzes the electroreduction of carbon dioxide into hydrocarbon fuels. Energy Environ Sci 3: 1311-1315.

[36] Rossmeisl J, Qu ZW, Zhu H, Kroes GJ, Nørskov JK (2007) Electrolysis of water on oxide surfaces. J Electroanal Chem 607: 83-89. 
Figures

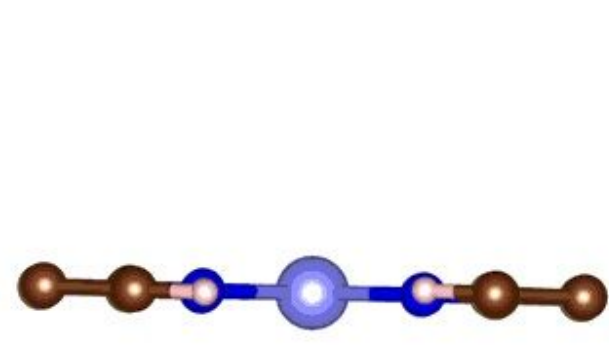

(a) Co-TCNQ

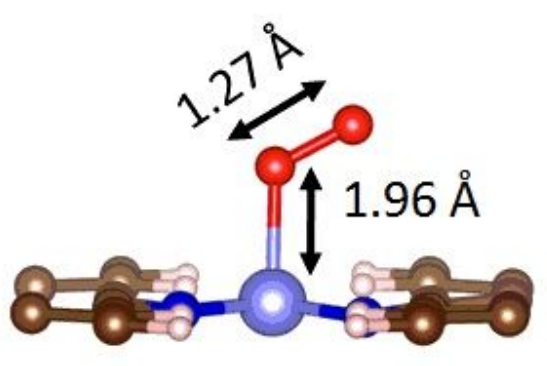

(b) Co-TCNQ-OO*

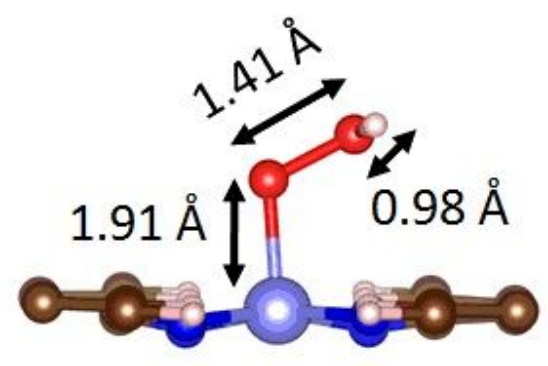

(c) Co-TCNQ-OOH*

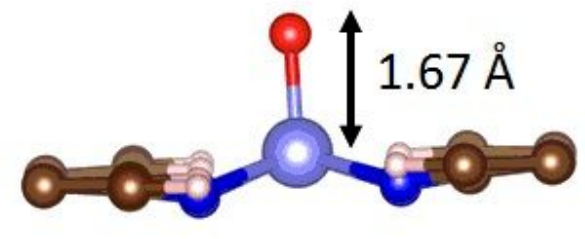

(d) Co-TCNQ-O*

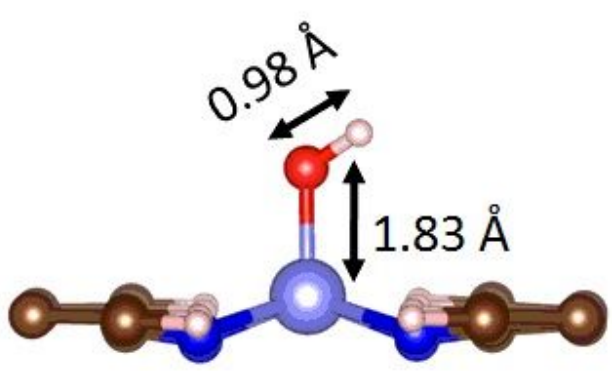

(e) Co-TCNQ-OH*

Figure 1

Optimized geometries of Co-TCNQ structures under different ORR steps.
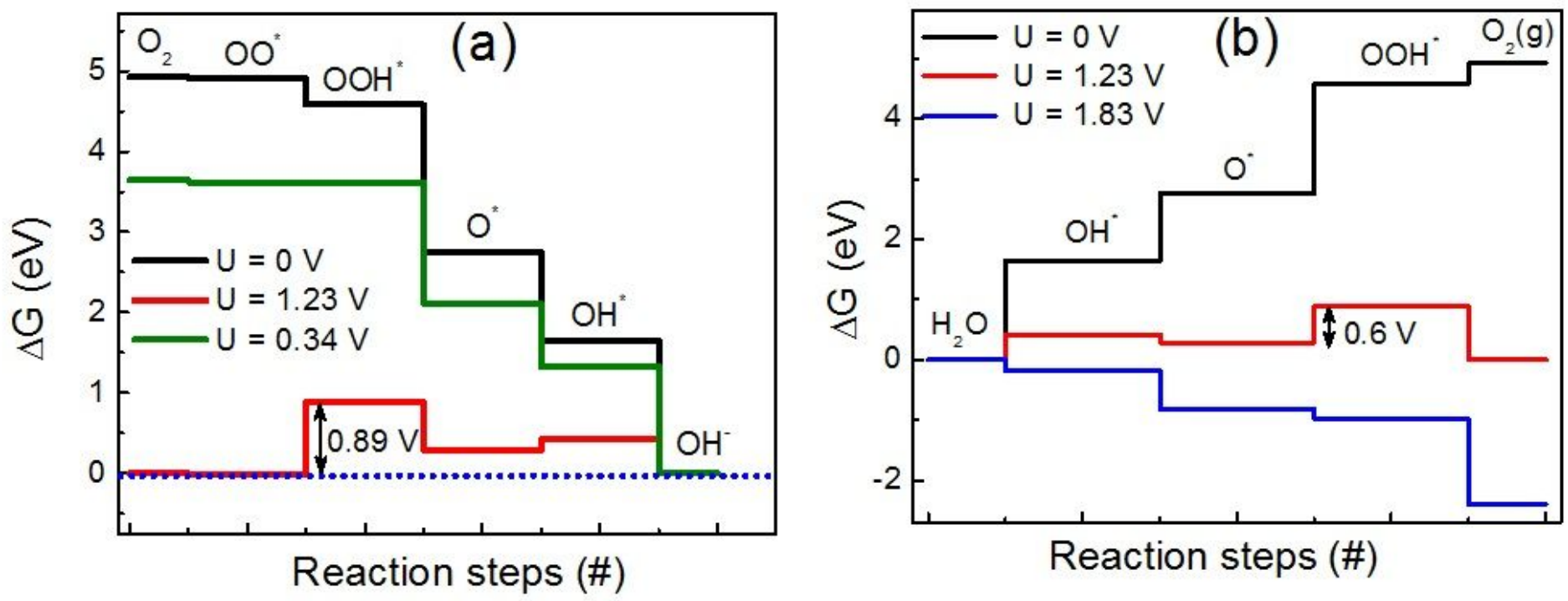

Figure 2 
Gibbs free energy profile for Co-TCNQ catalyst in acidic medium $(\mathrm{pH}=0)$ during $(\mathrm{a})$ ORR and $(\mathrm{b})$ OER.
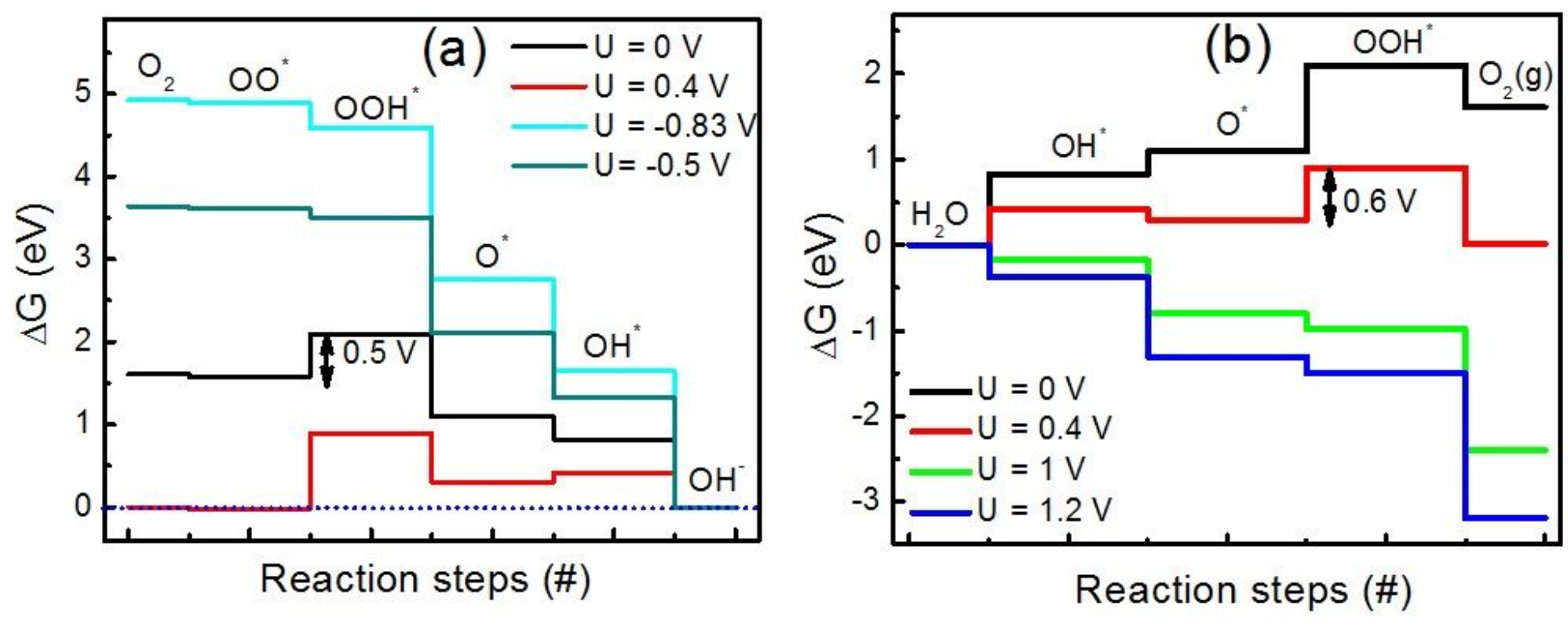

Figure 3

Gibbs free energy profile for Co-TCNQ catalyst in alkaline medium $(\mathrm{pH}=14)$ during $(\mathrm{a})$ ORR and $(\mathrm{b})$ OER. 


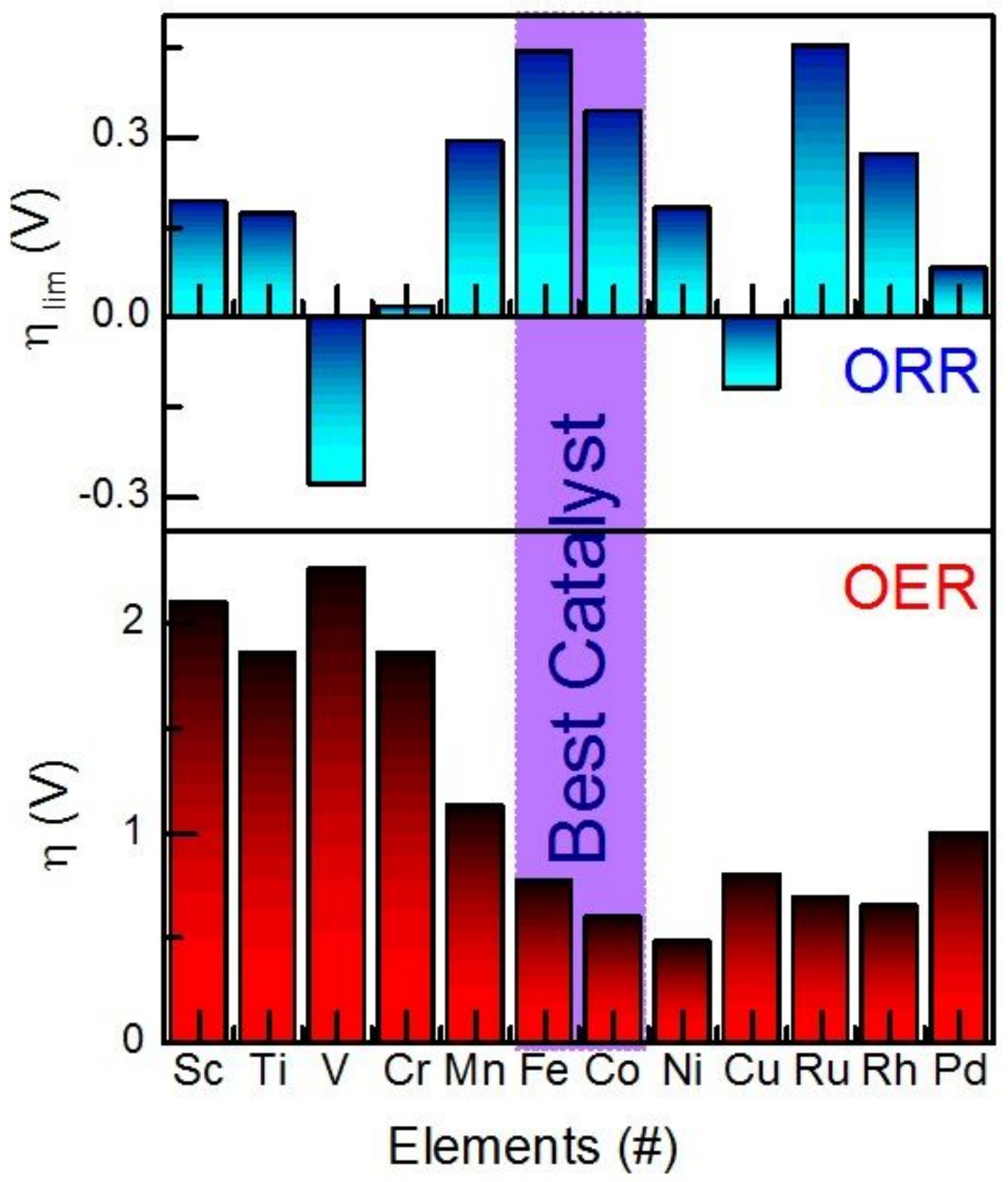

Figure 4

Bi-functional catalytic performances of various TM-TCNQ monolayer. The limiting potential ( $\eta$ lim) towards ORR (upper panel) and overpotential ( $\eta$ ) towards OER (lower panel) has been plotted for different TM-TCNQ monolayer. The best performance zone is marked by violet color. 


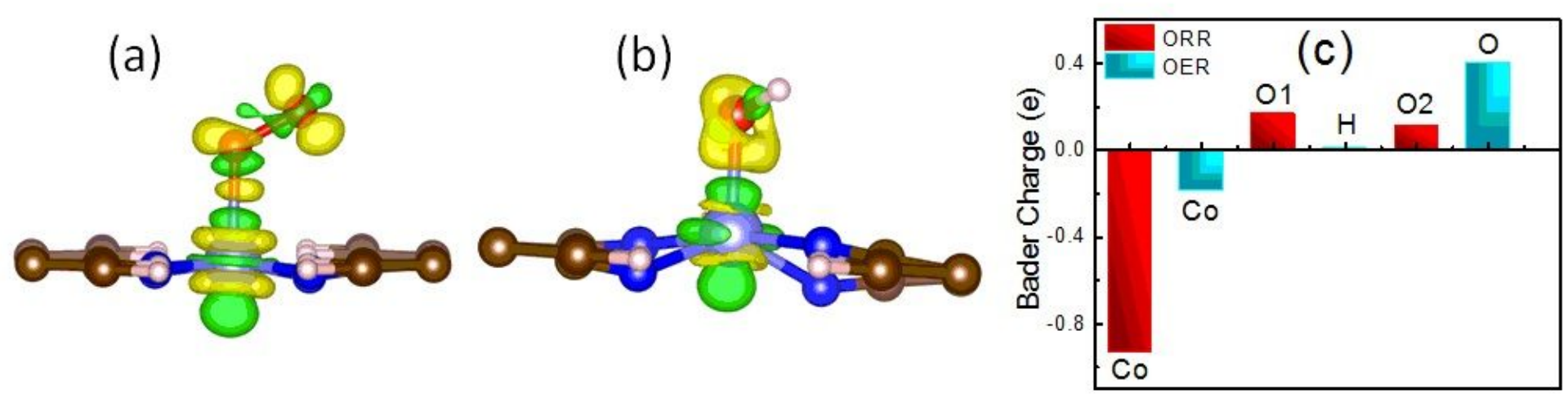

Figure 5

Charge re-arrangement and corresponding optimized geometries of Co-TCNQ monolayer during (a) 00* and (b) $\mathrm{OH}^{*}$ adsorbates. The yellow and green colors represent respectively the accumulation (negative) and depletion (positive) of charges. (c) Bader charge analysis for both ORR and OER.
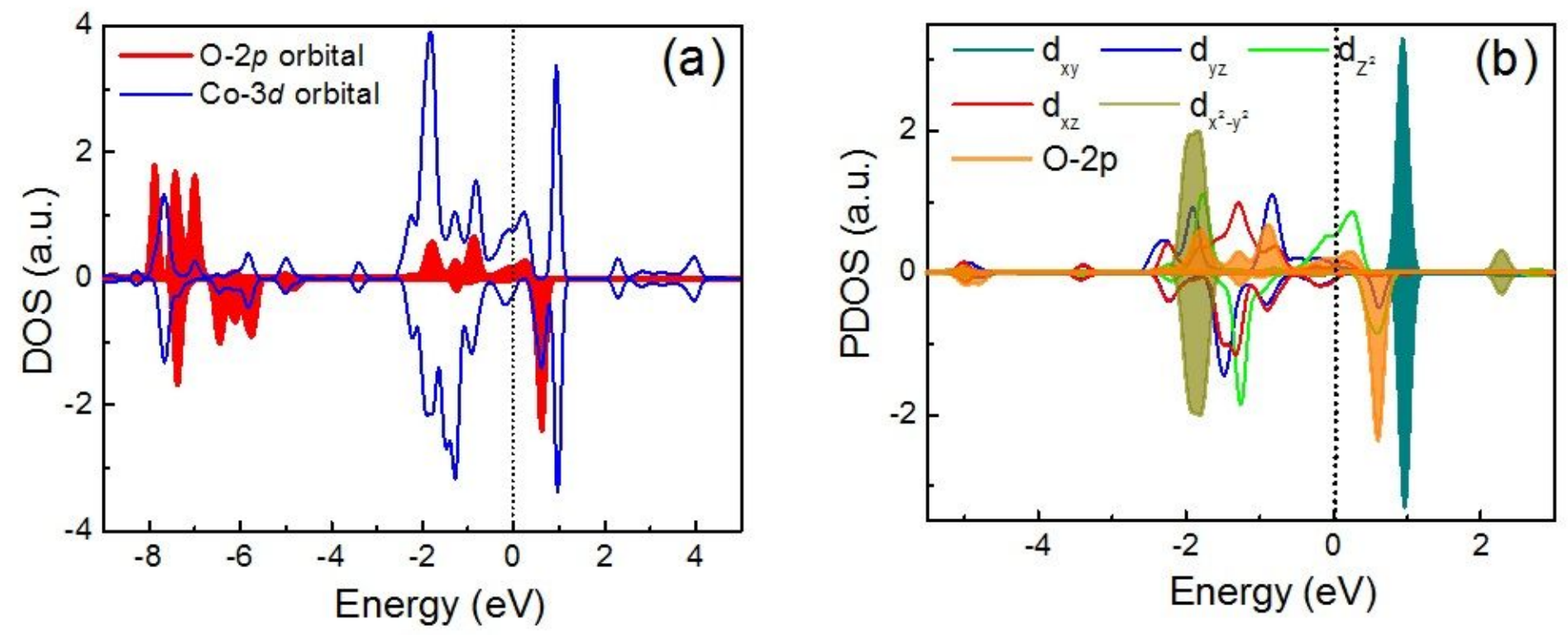

Figure 6

(a) Density of states (DOS) and hybridization between Co-3d and 0-2p orbitals. (b) PDOS of 0-2p orbital and different Co-3d orbitals. The dotted lines in the figures at $\mathrm{E}=0 \mathrm{eV}$ indicate the Fermi level. 